Service social

\title{
Le contrat en travail social : fondements éthiques et opérationnalité
}

\section{Cristina De Robertis}

Volume 43, numéro 3, 1994

Intervention individualisée et empowerment

URI : https://id.erudit.org/iderudit/706673ar

DOI : https://doi.org/10.7202/706673ar

Aller au sommaire du numéro

\section{Éditeur(s)}

École de service social de l'Université Laval

ISSN

1708-1734 (numérique)

Découvrir la revue

Citer cet article

De Robertis, C. (1994). Le contrat en travail social : fondements éthiques et opérationnalité. Service social, 43(3), 139-152. https://doi.org/10.7202/706673ar
Résumé de l'article

Alors que la pratique d'un contrat exigé par les dispositifs d'action sociale tend à se développer (aides au logement, jeunes majeurs, hébergement...), il n'est pas inutile de nous interroger sur les fondements du contrat en travail social et de nous rappeler les principes éthiques et l'intérêt opérationnel de cet outil. C'est le but de cet article qui se propose de définir ce qu'est le contrat, son intérêt et sa place dans la méthodologie professionnelle des travailleurs sociaux. Comme il s'agit d'une technique dont l'utilisation se révèle parfois peu aisée, nous traiterons des difficultés les plus courantes. Enfin, nous aborderons les fondements étiques du contrat en travail social et plus particulièrement les notions d'autonomie et d'acceptation du client, de confiance et d'engagement réciproques. 


\section{Le contrat en travail social : fondements éthiques et opérationnalitét}

Cristina DE ROBERTIS

Assistante de service social Diplômée de l'École de service social Université de Montevideo - Uruguay

Le contrat est un outil d'intervention utilisé en travail social depuis environ vingt ans. C'est donc une technique relativement récente, mais qui a déjà fait ses preuves.

En France, le contrat s'est frayé une voie dans la méthodologie professionnelle de manière progressive et continue. Les premières expériences d'utilisation ont été diffusées par Aide à Toute Détresse (A.T.D. Quart Monde) en 1977. Cette association utilisait systématiquement un contrat écrit avec les familles relogées temporairement en cité de promotion familiale. Ces contrats, d'une durée de six mois, étaient renouvelables et les termes pouvaient en être modifiés.

Depuis, I'utilisation du contrat s'est multipliée dans différents services, soit de manière ponctuelle et expérimentale (polyvalence,

* Cet article reprend la communication présentée au $48^{\mathrm{e}}$ congrès de l'Association nationale des assistants de service social: "Mandat, contrat, évaluation, quelle légitimité pour quel travail social ? », qui s'est tenu du 22 au 24 novembre 1993 à Paris. 
mandat judiciaire pour la protection de l'enfance et l'adolescence en danger, etc.), soit de manière plus institutionnalisée (Aide sociale à l'enfance). Mais c'est certainement le Revenu minimum d'insertion qui a donné à cette technique ses lettres de noblesse et sa légitimité.

En effet, la loi sur le RMI (Nº8-1088 du 1988-12-01) prévoit la passation d'un «contrat d'insertion» dans «les trois mois qui suivent la mise en paiement de l'allocation». Ce contrat, élaboré par le travailleur social avec le bénéficiaire, sera présenté à la commission locale $\mathrm{d}^{\prime}$ insertion (CLI) pour acceptation et signature. Ainsi, le contrat lie le bénéficiaire et la CLI. Le travailleur social est un intermédiaire, un "marginal sécant» pour reprendre l'expression de Michel Crozier.

Or, malgré l'esprit et la lettre de la loi, nous assistons souvent à une transformation des usages: d'une part, le contrat devient une procédure administrative, car il est requis pour percevoir l'allocation; $d^{\prime}$ 'autre part, les offres sociales d'insertion sont trop peu nombreuses et ne peuvent répondre à la demande d'emploi de nombreux chômeurs. Les exigences administratives (formulaire d'identification et de contrat $d^{\prime}$ insertion identiques pour toute la France) et les statistiques quantitatives (pourcentage d'allocataires ayant un contrat signé), malgré leur intérêt pour une évaluation nationale du dispositif RMI, font parfois perdre de vue l'objectif professionnel d'aide à la personne.

Alors que la pratique d'un contrat exigé par les dispositifs $\mathrm{d}^{\prime}$ action sociale tend à se développer (aides au logement, jeunes majeurs, hébergement...), il n'est pas inutile de nous interroger sur les fondements du contrat en travail social et de nous rappeler les principes éthiques et l'intérêt opérationnel de cet outil. C'est le but de cet article qui se propose de définir ce qu'est un contrat, son intérêt et sa place dans la méthodologie professionnelle des travailleurs sociaux. Comme il s'agit d'une technique dont l'utilisation se révèle parfois peu aisée, nous traiterons des difficultés les plus courantes: celles liées au client lui-même ou à sa situation externe, celles liées au travailleur social et celles liées aux institutions sociales. Enfin, nous aborderons les fondements éthiques du contrat en travail social et plus particulièrement les notions d'autonomie et d'acceptation du client, de confiance et d'engagement réciproques.

\section{DÉFINITION}

Mais qu'est-ce qu'un contrat? Le mot vient du latin contractus (de contrahere, "prendre engagement ») et l'idée est celle de convention, pacte, accord. C'est l'accord par lequel une ou plusieurs personnes 
s'obligent, envers une ou plusieurs autres, à faire, à donner ou à ne pas faire une chose déterminée. Le contrat a une signification juridique et économique; il s'agit de transactions de biens ou d'argent, telles que le contrat de vente, le contrat de travail, le contrat de bail.

Pour qu'un contrat soit valable au regard de la loi, il doit remplir certaines conditions:

- présence des parties ou, à défaut, présence d'une représentation reconnue (parents pour les mineurs, tuteurs pour les incapables majeurs, représentant désigné par l'intéressé) ;

- consentement des parties; les parties doivent être d'accord et capables de contracter librement. L'erreur, le dol ou la violence sont des vices au consentement et provoquent l'annulation du contrat;

- l'objet du contrat, la chose sur laquelle les parties s'obligent, doit être déterminé, possible, licite et en accord avec la morale et les bonnes mœurs.

En travail social le contrat constitue un accord entre trois parties: I'institution d'action sociale, le travailleur social et l'usager. Cet accord s'inscrit dans un processus méthodologique qui comporte différentes étapes: la phase initiale débute par la prise de contact avec le client, la phase finale par la clôture de l'intervention. Au cours de ce processus, le contrat s'élabore après la phase d'évaluation opérationnelle (diagnostic); c'est le moment de la confrontation et de la négociation des projets en présence, afin de construire un projet commun sur une base d'accord réciproque. II ne peut s'établir que dans le cadre d'une relation d'aide, car sans confiance, sans accord, point de contrat, point de relation d'aide. L'aide forcée ou imposée est perçue par le client comme une intrusion, comme une humiliation, et elle devient alors totalement inefficace.

Le contrat entre le travailleur social et le client stipule leur accord quant aux objectifs à atteindre, à la formulation des attentes réciproques, de même que le temps, le lieu, la fréquence des rencontres. Il s'agit donc d'une formalisation précise et explicite de ce qui va être entrepris ensemble afin d'améliorer la situation de l'usager, la formulation d'un accord commun.

\section{INTÉRÊT DU CONTRAT}

Pour quelles raisons formalise-t-on ainsi la relation d'aide entre le travailleur social et le client? Qu'apporte le contrat à l'intervention en travail social, quelle est son utilité? À quoi sert-il? 
Tout d'abord, le contrat apporte une direction et focalise les activités du travailleur social et du client sur des points précis préalablement définis. Cette focalisation sur des objectifs à atteindre favorise la mobilisation en commun des énergies et apporte un sentiment de sécurité et de confiance: on sait où l'on veut aller.

Ensuite, le contrat implique la reconnaissance du client comme responsable de sa vie, comme adulte capable. Il réaffirme l'importance de la participation active de l'usager dans la résolution de ses propres problèmes, le situe en tant qu'acteur, en tant que sujet et non point en tant qu'objet d'aide. Il est ainsi intimement associé au projet qui concerne son avenir et dont il reste le principal maître d'œuvre, le principal acteur.

De plus, le contrat - du fait du travail de clarification qu'il implique - aide à réduire les écarts entre les attentes du client et celles du travailleur social. Il facilite l'engagement sur des bases plus claires et réalistes. Cette réduction des écarts et cette clarification des attentes ne changent en rien la différence de position entre le travailleur social et le client, car le contrat ne rend pas la relation "égalitaire», elle permet d'établir une relation sur des bases explicites sans toutefois effacer les rôles et pouvoirs différents de chacun.

Enfin, le contrat apporte une base d'évaluation des résultats et facilite la mesure de la progression vers les objectifs définis. En effet, il n'y a pas d'évaluation des résultats sans définition précise et préalable des buts à atteindre, et, du fait de cet accord explicite, le contrat fournit des repères pour mesurer le chemin parcouru.

\section{UTILISATION DU CONTRAT DANS NOTRE MÉTHODOLOGIE PROFESSIONNELLE}

Rappelons tout d'abord que le contrat est un moyen, un outil de travail. Ce n'est pas une fin en soi, ni une panacée ou un remède miracle. II ne s'agit pas de passer un contrat pour le contrat en soi ; il s'agit d'employer les moyens $d^{\prime}$ aide les plus utiles et les plus efficaces par rapport au client.

Pour que le contrat garde sa véritable fonction d'aide, il doit être souple et flexible, il doit pouvoir être révisé à tout moment et renégocié périodiquement pour le réajuster. En effet, il est centré sur le bénéficiaire, sur ses besoins, ses aspirations, ses capacités.

Ainsi, le contrat devient un véritable outil et fait partie de notre «boîte à outils». Il nous sert à : 
- structurer un cadre de travail, établir des objectifs opérationnels, un plan de travail, une répartition des tâches, et ce cadre a un effet sécurisant ;

- dynamiser et mobiliser les énergies, car on sait quel est le but à atteindre, quels sont les moyens à employer (ensemble le travailleur social et l'usager vont se mobiliser). C'est une tout autre attitude que celle de I'attente passive («que I'assistante sociale trouve une solution ou que l'usager donne de ses nouvelles»);

- obtenir des succès rapides, car la plus petite réussite est source d'espoir, de valorisation, de légitimité. Redonner confiance dans ses possibilités et espoir dans l'avenir est la clé de voûte de la pédagogie de la réussite. Le succès décuple l'énergie et le dynamisme. L'échec, par contre, renforce la passivité, la crainte et détruit l'auto-estime.

\section{ÉLABORATION ET FORME DU CONTRAT}

Avant même la construction du contrat, une étape préliminaire est indispensable; c'est le moment de la prise de contact, du démarrage de la relation. $C^{\prime}$ est le temps de la rencontre.

Au cours de cette phase, il s'agit de se connaître, d'explorer les attentes réciproques, de clarifier les rôles et la position de chacun. Cette connaissance permettra progressivement d'asseoir une base de confiance indispensable à la construction du contrat.

Nombreux sont les travailleurs sociaux qui, en France, assurent utiliser la technique du contrat uniquement avec des personnes déjà suivies, déjà connues parfois de longue date.

Toute cette phase préliminaire conduit le travailleur social et le client à approfondir leur compréhension de la situation, à mieux cerner les caractéristiques du problème et à établir ensemble une évaluation des capacités et des ressources existantes.

L'évaluation des capacités et des ressources concerne la situation du client; elle permettra au client et au travailleur social de se mettre d'accord sur une même définition du problème et des objectifs à poursuivre.

Aux capacités propres du client (physiques, relationnelles, intellectuelles, capacités à agir) s'ajoute l'évaluation des ressources externes. Parmi celles-ci, il y a celles issues de son réseau primaire (famille, amis, voisinage) et celles liées au réseau secondaire, 
comportant les services, institutions, groupes qui existent dans la société locale et auxquels on peut avoir recours.

Lors de la négociation du contrat avec l'usager, tout un processus de travail nous amène à :

- clarifier les problèmes et les aspirations (les définir, se mettre $d^{\prime}$ accord sur un choix de priorités, confronter les désirs du client et ceux du travailleur social);

- établir des objectifs communs (définir les objectifs de changement, c'est-à-dire quelle situation atteindre, quelles transformations produire);

- élaborer un plan de travail (se mettre d'accord sur ce qu'on va faire, la façon de procéder, la répartition des tâches, la durée, la structuration dans le temps);

- formaliser le contrat: toute une gamme de possibilités s'offrent à nous, de la plus formelle (contrat écrit, signature de chaque partie, parfois lors d'une rencontre avec un caractère solennel) à la plus informelle (contrat oral).

La mise en œuvre du contrat nécessite des rencontres régulières pour assurer un accompagnement et la possibilité de réajuster les termes du contrat, afin de lui conserver toute son utilité, c'est-à-dire dynamiser la démarche de changement et la rendre plus efficace.

\section{LES DIFFICULTÉS D'UTILISATION DE CETTE TECHNIQUE}

Même si le contrat s'avère une technique utile et positive dans l'exercice professionnel des travailleurs sociaux, certains écueils existent, certaines difficultés se font jour dans son application.

Nous nous attacherons dans ce paragraphe à souligner certaines de ces difficultés sans prétendre en dresser une liste exhaustive. Nous les classerons en quatre types: les difficultés liées au client lui-même, celles qui ont pour origine la situation externe au client, celles venant du travailleur social et celles liées aux institutions.

\section{a) Les difficultés liées au client}

- Le client n'est pas demandeur

Lorsque le client est demandeur et actif, lorsqu'il sait ce qu'il souhaite et qu'il est capable de se mobiliser pour l'obtenir, I'utilisation du contrat renforce ses dynamismes. 
Par contre, il est plus difficile de passer un contrat lorsque le client n'est pas demandeur, lorsqu'il est peu disposé à accepter l'aide du travailleur social, lorsqu'il ne sait pas quelle sorte de problèmes il pourrait tenter de résoudre avec son soutien.

- Le client est incapable de s'organiser dans le temps

Avec certaines personnes incapables de s'organiser dans le temps, de prévoir l'avenir à moyen terme, formaliser un contrat peut se révéler très difficile. De même, si le client est très instable, changeant, s'il ne sait pas se tracer une ligne de conduite et $s^{\prime} y$ tenir, la mise en place d'un contrat sera très aléatoire.

\section{b) Les difficultés liées à la situation externe}

- Pauvreté des réseaux primaires

Un certain nombre d'usagers du travail social ont perdu, ou $\mathrm{n}^{\prime}$ ont jamais eu, un réseau de relations familiales et de proximité suffisamment fort et soutenant. La mobilité géographique et la vie urbaine ont accéléré la dislocation des liens affectifs, les ruptures et l'isolement. Ces personnes aux réseaux primaires faibles, peu nombreux et peu diversifiés sont plus exposées que les autres aux problèmes auxquels « on ne peut pas faire face». En effet, ce sont la famille, les amis proches, les voisins qui, lorsqu'ils existent dans des relations qui comptent, constituent les principales ressources matérielles et affectives immédiatement disponibles.

- Insuffisance des ressources disponibles

Lorsque le problème principal du client a trait à des difficultés sociales qui touchent un nombre important de personnes d'un même lieu (chômage à la suite d'une fermeture d'usine, personnes mal logées après expulsion, personnes qui ne peuvent supporter le coût d'un loyer, problème de garde d'enfants dans les familles monoparentales, etc.), il n'est plus le seul concerné, mais fait partie d'un ensemble de personnes qui font face à des problèmes similaires. Dans ces cas, les possibilités offertes par la société pour résoudre ces difficultés peuvent être insuffisantes, inefficaces ou inexistantes. Il est fréquent, en effet, que la législation sociale et les ressources disponibles ne suivent qu'avec bien du retard la manifestation de certains problèmes sociaux plus ou moins collectifs. 


\section{c) Les difficultés liées aux institutions}

Les organismes d'action sociale, et notamment ceux chargés des nouveaux dispositifs territoriaux et d'insertion, utilisent le contrat comme mode de relation avec les usagers. II n'y a pas un service, une administration, une équipe de travailleurs sociaux qui ne réfléchisse à la meilleure manière de "contractualiser» la relation avec les usagers. Alors qu'il y a encore dix ans cette pratique était vue comme une curiosité, aujourd'hui c'est plutôt I'engouement. Le RMI est passé par là et a apporté au contrat un cadre juridique et réglementaire très précis, lui accordant ses lettres de noblesse et sa légitimité.

Nous ne pouvons que nous réjouir de cette évolution qui tend à considérer l'usager comme acteur et partenaire principal dans le changement de sa situation, et non comme simple destinataire $d^{\prime}$ aides et services divers.

Toutefois, nous percevons aussi une série de contradictions, "d'effets pervers", dans l'usage qui est fait du contrat par certaines institutions; elles nous semblent pouvoir hypothéquer l'avenir si nous n'y prenons garde à temps. Car certains usages et pratiques dénaturent profondément le sens du contrat en tant qu'outil au service des usagers.

Le premier effet pervers est celui d'utiliser le contrat comme une fin en soi. Le contrat devient le but, l'objet valorisé, on a fait du bon travail quand on a passé un contrat... et le plus vite possible... Le contrat n'est plus un outil, un moyen de dynamisation, de négociation, de confrontation entre le travailleur social et le client. Parfois, c'est la pression institutionnelle qui s'exercera sur le travailleur social: "Comment? Vous n'avez pas encore conclu le contrat avec $M^{\text {me }} X$ ? » Et le travailleur social de se faire tout petit, face au regard réprobateur de ses chefs ou de ses collègues.

Le contrat peut aussi devenir une forme de pression sur I'usager: "Vous bénéficierez de telle allocation, de tel secours, mais... seulement si vous signez un contrat acceptant de...». Le client a peu de moyens de s'y opposer ou même de protester. II dira oui, mais le contrat sera suivi de quel effet? Parfois, il dira non, et alors il sera privé d'un soutien dont il a réellement besoin.

Une forme de contrat se développe de plus en plus, le «contrat standard». Ce «contrat» consiste en un imprimé 
identique pour tous les usagers, où figurent les droits et obligations auxquels ils doivent s'astreindre s'ils veulent bénéficier... d'un "placement jeunes majeurs", d'une allocation mensuelle, $d^{\prime}$ une place en centre $d^{\prime}$ 'hébergement ou en maison de retraite, etc. Ces "contrats» sont plutôt des formulaires administratifs ou des règlements intérieurs. Ils ne tiennent absolument pas compte de la situation individuelle ou familiale de la personne, ni de son projet personnel. Ils ne peuvent donc être considérés comme un contrat au sens professionnel que nous utilisons ici. À la rigueur, ils constituent le cadre institutionnel et légal au sein duquel un véritable contrat entre travailleur social et client peut se construire.

Certains de ces usages institutionnels s'imposant aux clients et aux travailleurs sociaux, le danger est de transformer cet outil en un papier inerte, sans signification, sans vie, de dénaturer l'essence même du contrat qui est la recherche d'un accord, d'un partage, d'une nouvelle dynamique et d'un nouvel espoir.

\section{d) Les difficultés liées au travailleur social}

- Le poids de la routine

Devant une nouvelle technique, une manière de faire différente, le travailleur social peut, lui aussi, être confronté à ses propres résistances au changement. Faire "comme d'habitude" est plus simple, nécessite moins d'effort, donne des résultats satisfaisants, ou au moins passables. C'est la routine qui s'installe: le travail un peu "à la chaîne", celui qu'on connaît bien, celui qu'on sait faire. À quoi bon essayer autre chose? Pourquoi réfléchir à un contrat, alors que jusqu'à présent on n'en a jamais fait? Attendons donc que la loi l'impose... II faut tout d'abord dépasser la barrière de l'inertie naturelle.

- Le réflexe "distributeur de réponses »

La plupart des travailleurs sociaux connaissent bien les ressources sociales et institutionnelles disponibles dans leur secteur et dans leur domaine d'activité. Qu'il s'agisse de prestations légales, de secours financiers ou en nature, $d^{\prime}$ 'institutions (crèches, foyers, hôpitaux, etc.), leur compétence en matière de ressources sociales $n^{\prime}$ est plus à démontrer. En outre, ils savent où et comment obtenir les renseignements sur les ressources avec lesquelles ils sont moins familiarisés (secteur ou type de clientèle non habituelle). 
Pourtant, cette compétence peut leur jouer un mauvais tour lorsqu'il s'agit d'élaborer un contrat avec l'usager. Trop focalisé sur la satisfaction rapide des besoins décelés dans la situation du client, le travailleur social propose des solutions en fonction des ressources existantes et de ce qu'il connaît bien comme services, prestations, circuits institutionnels, législation sociale, etc. La rapidité et l'efficacité semblent excellentes. Le travailleur social est content, le client aussi, mais... jusqu'à la prochaine fois.

Le contrat entre le travailleur social et le client se trouve dénaturé : au lieu d'être centré sur le client et sa situation (ses besoins, ses capacités, ses potentialités), il est centré sur les ressources sociales disponibles, même si elles ne correspondent pas exactement aux besoins du client.

Pour éviter que son rôle même ne se trouve ainsi transformé, le travailleur social aura à trouver le bon équilibre entre:

- la connaissance la plus complète possible des ressources sociales existantes,

- I'utilisation des ressources adaptées à la situation du client,

- I'engagement dans une relation personnalisée qui apporte au client reconnaissance, valorisation, estime de soi et autonomie.

\section{LES FONDEMENTS ÉTHIQUES}

Quels sont les fondements éthiques de la démarche contractuelle, quelles valeurs guident I'utilisation de cette technique? Les valeurs qui sous-tendent le contrat font référence au respect de l'autonomie du client, à son acceptation, à l'instauration d'une confiance réciproque et à la reconnaissance des compétences et des capacités du client.

\section{Autonomie}

Le contrat reconnaît la capacité d'autonomie du client, car, au-delà de sa situation de dépendance provisoire, celui-ci garde l'entière responsabilité de sa vie. II garde "le droit de vivre sa propre vie et de participer activement aux décisions et aux choix au cours du processus » d'intervention sociale. La reconnaissance de l'autonomie du client va donc de pair avec sa participation active à tout ce qui le concerne, sa vie présente et son avenir. 
Le contrat va stimuler cette participation active à tous les niveaux: réflexion sur la situation, projets d'avenir, décisions à prendre. Dès le début de la relation avec le travailleur social, le client sera sollicité pour prendre les décisions qui le concernent, qui concernent sa vie. Ensemble, travailleur social et client réfléchiront aux décisions à prendre, ils en discuteront et les élaboreront. Le travailleur social facilitera l'exploration des différentes possibilités ou alternatives et la prise en compte des avantages et inconvénients de chacune, mais la décision à prendre reste de la compétence et du ressort exclusifs de l'usager.

Le travailleur social doit reconnaître la capacité d'autodétermination du client et ne jamais empiéter sur ce droit fondamental de décider de sa propre vie.

\section{Acceptation}

Accepter l'autre, c'est reconnaître son droit à la différence, son droit à être un individu unique, son droit de penser et d'agir différemment de nous-même et de tous les êtres humains. L'acceptation de l'autre prend donc sa source dans la reconnaissance de sa différence, de son unicité.

Or, les personnes sont aussi semblables les unes aux autres: elles ont les mêmes besoins de base (nourriture, toit, sommeil) et les mêmes besoins d'affection, d'appartenance, de reconnaissance; elles ont aussi les mêmes droits et les mêmes obligations. Ainsi, quelle que soit sa situation présente, le client est une personne à la fois unique et différente de toutes les autres, et en même temps semblable à tous les êtres humains. Il est revêtu de la même dignité, porteur des mêmes droits.

Le client est accepté lorsque le travailleur social est capable de le comprendre tel qu'il est et de respecter son intégrité et sa dignité en tant qu'être humain semblable à lui-même.

L'acceptation de l'autre fait référence à l'idée de respect. Le respect du client comprend le respect de ses options, de ses croyances, de sa culture, de ses normes d'existence, de ses choix de vie. II comporte une attitude de non-jugement, c'est-à-dire que le travailleur social ne porte aucun jugement de valeur en termes de bon ou mauvais, d'innocent ou coupable sur la situation du client. Il se garde d'appliquer sa propre échelle de valeurs au client; au contraire, il s'efforce de l'aborder sans idées préconçues et de découvrir sa réalité, de comprendre son histoire, sa pensée, ses sentiments. 


\section{Confiance réciproque}

L'acceptation de I'autre en tant que personne est le terreau dont se nourrit la confiance. Les travailleurs sociaux parlent souvent de "relation de confiance» et cette expression n'est pas toujours comprise par les interlocuteurs néophytes.

Le Code de déontologie de I'A.N.A.S. stipule une série d'obligations générales des professionnels, dont: "Le respect de la personne fonde, en toutes circonstances, I'attitude de l'assistant de Service social. II implique, en particulier, la recherche constante d'une relation personnelle de confiance avec le client. » La recherche d'une relation de confiance fait partie des devoirs du professionnel et, sans confiance, il n'y a pas de relation contractuelle possible.

Lorsque le client se sent accepté tel qu'il est, il n'a plus besoin de «jouer un rôle», de faire semblant de se conformer à l'image qu'il croit que le travailleur social attend de lui.

Ce qui est alors ressenti par le client, c'est que le travailleur social lui fait confiance... Confiance en ses capacités, en ses compétences pour trouver les bonnes solutions, pour prendre les décisions adéquates. L'assurance de la confiance du travailleur social contribue à sa valorisation personnelle.

Nous voyons ainsi que la confiance est réciproque, qu'elle se crée et se construit dans un climat relationnel chaleureux, ouvert et bienveillant, qu'elle n'est pas gagnée d'avance. La confiance du client est indispensable au travailleur social, car sans elle aucun processus de changement constructif ne pourra se produire. De même, la confiance du travailleur social dans les forces et capacités du client doit être à toute épreuve.

Pour conserver la confiance des usagers, pour leur garantir le respect de leur vie privée, le secret professionnel fait obligation aux assistants de service social de ne pas divulguer les faits confidentiels appris dans l'exercice de la profession. Le secret professionnel protège la vie privée de l'usager, il le met à l'abri de toute utilisation des confidences qu'il a pu nous faire. Ce n'est nullement une prérogative, un privilège ou un alibi de notre profession, mais bien la garantie, pour le client, de la protection de sa vie privée et de la confiance qu'il peut nous accorder.

\section{Engagement réciproque}

De plus, le contrat oblige à un engagement des différentes parties. Ce $\mathrm{n}^{\prime}$ est pas un engagement unilatéral, mais bien un engagement 
réciproque. L'usager - "citoyen» et porteur de droits - a aussi des obligations à respecter, mais il n'est pas seul dans cet engagement: le travailleur social, l'institution, la société sont eux aussi engagés.

Prenons I'exemple du contrat RMI. II s'élabore entre le bénéficiaire et le travailleur social. Ce dernier assume un rôle de médiation et ensuite assure le suivi. Le contrat, une fois défini, sera accepté et signé par le président de la CLI (Commission locale d'insertion) qui représente la société. La société, par l'intermédiaire de la CLI, est donc engagée elle aussi dans le contrat ainsi établi. L'allocataire fait alors partie d'un réseau de personnes et d'instances engagées par ce contrat. II n'est plus isolé, il occupe une place dans ce réseau. Le contrat d'insertion participe ainsi à la création du lien social, il formalise pour l'usager une place dans la société.

\section{CONCLUSION}

Quel sens attribuer à l'utilisation de la technique du contrat en travail social?

Notre premier devoir est de prendre en considération le bénéficiaire en tant que personne, en tant qu'être humain, sujet et acteur de sa vie. Nous gardons envers lui une vision globale qui tient compte de la complexité de sa situation, des facettes multiples de son histoire, de son présent et de son devenir. Nous ne pouvons pas découper sa situation en tranches, nous cherchons à rendre compte de la cohérence holistique de son itinéraire, et le contrat représente un moment dans ce devenir.

Ainsi focalisé sur la personne et intimement lié à son cheminement de vie, le contrat devient un moyen, parmi d'autres, de favoriser I'accès :

- aux droits et à la citoyenneté,

- à la parole, parole exprimée, parole écoutée, parole prise en compte,

- à une place reconnue dans la société,

- à un pouvoir social, c'est-à-dire la possibilité d'exister en tant que partie prenante d'une société et comptant pour elle.

$C^{\prime}$ est ce que les Canadiens appellent empowerment et qui, malgré la difficulté de trouver une traduction équivalente, signifie faire accéder l'usager à un pouvoir social où il puisse exercer pleinement ses prérogatives d'être humain vivant en société. 


\section{Références bibliographiques}

Colloque national 1986 (1988). Le contrat en sciences humaines dans les pratiques sociales et de santé. Rennes: Presses universitaires de Rennes, Annales cliniques et psychologiques.

CONSEIL SUPÉRIEUR EN TRAVAIL SOCIAL (1988). Rapport au ministre: l'intervention sociale d'intérêt collectif. La documentation française.

DAVID, M. et P. MAUROY (1982). La solidarité comme contrat et comme éthique. Paris: Berger-Levrault, Collection Mondes en devenir.

DE ROBERTIS, C. (1981). Méthodologie de l'intervention en travail social. Chapitre 6 - Le contrat avec le client. Paris: Centurion, Collection Socioguides.

DE ROBERTIS, C. (sous la direction de) (1993). Le contrat en travail social. Paris: Bayard Éditions, Collection Travail social.

DOUSTENS, R. et Y. PINÇON (1985). Le travailleur social aux mains nues. Mayenne: Imprimerie Copainville, Mayenne.

DU RANQUET, M. (1983). Recherches en case work : I'efficacité et sa mesure en service social. Toulouse: Privat Edisem.

DU RANQUET, M. (1989). L'approche en service social. Centurion, Collection Travail social.

HEAP, K. (1987). La pratique du travail social avec des groupes. Paris: ESF.

HouARD, A. et F. Duffetelle (1985). Deux cantonniers en service social: ils utilisent d'abord les ressources de gens. Paris: CTNERHI.

Informations sociales (1992). "Les contrats d'aujourd'hui », n 19, Paris.

Lejeune, R. (1988). Réussir l'insertion: revenu minimum. Paris: Syros, Alternatives.

Ministère de la Solidarité, de la SAnté et de la Protection SOCiale DAS (1989). Le contrat avec les usagers dans la pratique professionnelle des travailleurs sociaux, Document de réflexion, Ille-de-France: CREAI, janvier.

PINÇON, Y. (1990). "L'intervention de réseaux, un projet d'action, des outils d'analyse », Cahiers de l'ARPE, n 3, Le naissement, Le Bourgneuf-la-Forêt.

REID, W. et L. EPSTEIN (1985). Travail par objectifs en service social. Toulouse: Privat-Edisem.

Rousseau, J.-J. (1966). Du contrat social. Paris: Flammarion.

SHULMAN, L. (1976). Une technique de travail social avec des groupes; le modèle de médiation. Paris: ESF, Collection Pratiques sociales.

THÉVENET, A. (1989). RMI, Théorie et pratique, Centurion, Collection Travail social.

TROUSSARD, F., G. POUSSIN et al. (1984). L'acte psychologique dans le champ de l'ASE - De l'analyse du geste à l'intelligence d'un contrat avec I'usager... avec l'employeur. Journées de perfectionnement ANPASE, Canteleu: Édition ANPASE.

VERDIER, P. (1993). Le guide de l'Aide sociale à l'enfance. Bayard Éditions, Collection Travail social.

WRESINSKI, J. (1987). "Rapport sur la grande pauvreté et précarité économique et sociale», Conseil économique et social, Journal officiel du 28 février 1987. 